\title{
Determining the range of magnetic interactions from the relations between magnon eigenvalues at high-symmetry $\mathrm{k}$ points
}

\section{Di Wang}

Nanjing University

Jihai Yu

Nanjing University

Feng Tang

Nanjing University

Yuan Li

Peking University https://orcid.org/0000-0002-2747-0649

Xiangang Wan ( $\nabla$ xgwan@nju.edu.cn )

Nanjing University https://orcid.org/0000-0001-7727-4980

\section{Article}

Keywords: Magnetic exchange interactions, MEls, magnon energies (SSME), high-symmetry k points

Posted Date: April 8th, 2021

DOI: https://doi.org/10.21203/rs.3.rs-334283/v1

License: (c) (1) This work is licensed under a Creative Commons Attribution 4.0 International License. Read Full License 


\title{
Determining the range of magnetic interactions from the relations between magnon eigenvalues at high-symmetry $k$ points
}

\author{
Di Wang, ${ }^{1,2}$ Jihai Yu, ${ }^{1,2}$ Feng Tang, ${ }^{1,2}$ Yuan $\mathrm{Li}^{3,4}$ and Xiangang Wan ${ }^{1,2, *}$ \\ ${ }^{1}$ National Laboratory of Solid State Microstructures and School of Physics, Nanjing University, Nanjing 210093, China \\ ${ }^{2}$ Collaborative Innovation Center of Advanced Microstructures, Nanjing University, Nanjing 210093, China \\ ${ }^{3}$ International Center for Quantum Materials, School of Physics, Peking University, Beijing 100871, China \\ ${ }^{4}$ Collaborative Innovation Center of Quantum Matter, Beijing 100871, China
}

(Dated: March 16, 2021)

\begin{abstract}
Magnetic exchange interactions (MEIs) define networks of coupled magnetic moments and lead to a surprisingly rich variety of their magnetic properties. Typically MEIs can be estimated by fitting experimental results. But how many MEIs need to be included in the fitting process for a material is not clear a priori, which limits the results obtained by these conventional methods. In this paper, based on linear spin-wave theory but without performing matrix diagonalization, we show that for a general quadratic spin Hamiltonian, there is a simple relation between the Fourier transform of MEIs and the sum of square of magnon energies (SSME). We further show that according to the real-space distance range within which MEIs are considered relevant, one can obtain the corresponding relationships between SSME in momentum space. We also develop a theoretical tool for tabulating the rule about SSME. By directly utilizing these characteristics and the experimental magnon energies at only a few high-symmetry $k$ points in the Brillouin zone, one can obtain strong constraints about the range of exchange path beyond which MEIs can be safely neglected. Our methodology is also general applicable for other Hamiltonian with quadratic Fermi or Boson operators.
\end{abstract}

\section{INTRODUCTION}

As one of the oldest scientific topics, magnetism is still of great interest [1-4]. Magnetic materials had already been widely used in electromechanical and electronic devices, and its applications in information technology are also continuously growing [1-3]. Especially magnons, as the quanta of spin waves, have received more and more research attention over the past few decades $[5,6]$. As an elementary excitations of magnetic systems, magnons became an interesting platform for the study of general wave dynamics [7, 8], Bose-Einstein condensation of magnon [9-11] and so on. In addition, with the development of topological physics in the electron system, topology in magnon spectrum has also attracted significant interests [12-14], including topological magnon insulators [15, 16], magnonic Dirac semimetals [17-21] and Weyl semimetals [22-24]. Besides fundamental research, magnons have also attracted great attention for applications of information transport and processing [25-29]. Analogous to spintronics, the application of magnon are connected with the ability to carry, transport and process information. Potentially, the spins can be manipulated without current, thereby overcoming an important fundamental limitation of conventional electronic devices, the dissipation of energy due to Ohmic losses. Magnon spintronics is therefore an emerging field of modern magnetism, which has spurred significant advances towards computing application recently and is believed to deliver a number of breakthrough developments in the future [25-29]. In order to quantitatively understand the

* The corresponding author: xgwan@nju.edu.cn. rich phenomenon and wide applications in this highly interdisciplinary field, a microscopic magnetic model with proper parameters becomes extremely important.

Magnetic properties can be typically described by a quadratic spin Hamiltonian $H=\sum_{i, j} \mathbf{S}_{i} \cdot \mathbf{J}_{i j} \cdot \mathbf{S}_{j}=$ $\sum_{i, j} J_{i j}^{\alpha \beta} S_{i}^{\alpha} S_{j}^{\beta}$, where $\mathbf{J}_{i j}$ represents the magnetic exchange interaction (MEI) between the spin at $i$ site $\mathbf{S}_{i}$ and spin at $j$ site $\mathbf{S}_{j}$ as shown in the following Eq. (1). The sum should take over all possible exchange paths with sizable MEIs. However, it turns out that extracting quantitative $J_{i j}$ is a highly non-trivial task. By choosing the set of parameters that best fit the experimental results, such as temperature-dependent magnetization, magnetic susceptibility $\chi(T)$, magnetic excitation spectra $\omega(q)$ etc, one basically can obtain MEIs $J$ 's [1-4]. It is well known that the $J_{i j}$ usually decreases rapidly with increasing of $R_{i j}$, the distance between magnetic moment at $i$ and $j$ sites, and the $J$ 's with sufficient distance are believed to be negligible. Thus only a number of $J$ 's within a cut-off range $R_{\text {cut }}$ are needed to be considered. However, a priori knowledge about $R_{c u t}$ is unknown, while the number of MEI used to fit the experimental data obviously affect the obtained $J$ 's. This leads to the arbitrariness of fitting approach, consequently affected the accuracy of the estimated MEIs, and currently unambiguous fitting is basically impossible. For example, very similar inelastic neutron scattering (INS) experimental results can be fitted by considerably different MEI parameters [20, 21].

In addition to the above approach, theoretical calculations had also been used to evaluate the exchange interaction parameters [30-38]. A popular numerical method is to calculate the total energies of more than $N$ magnetic configurations and map them using a spin Hamiltonian to extract $N$ MEIs [30]. Unfortunately, this method also 
need to assume a cut-off range $R_{c u t}$, which again leads to the arbitrariness about the calculated MEIs. An alternative method is based on combining magnetic force theorem and linear-response approach [31-35]. Working in the momentum space, this method indeed does not suffer the problem about $R_{\text {cut }}$. However, the Coulomb interaction which had been incorporated by the parameter $U$ in first-principles calculations, usually play important role in magnetic systems $[39,40]$. Thus these theoretical MEIs strongly depend on the choice of the parameter $U$ $[39,40]$.

Symmetry imposes constraints about the magnetic model, and one can also use symmetry to check if two exchange paths with the same bond length have the same MEI. Unfortunately, this powerful theoretical method cannot provide any clue about $R_{\text {cut }}$. The general features, such as sum rule for the spectral weight of the spin correlation function [41] which requires accurate crosssection measurements over the entire Brillouin zone (BZ), also cannot predict the variation of MEIs over distance. Thus to explore possible $R_{c u t}$-related generic rules is a very important problem. Certain important subjects on magnetism, such as quantum spin liquids arising from exactly solvable models [42], novel properties from geometrically frustrated magnet [43], etc., explicitly requires small $R_{\text {cut }}$, hence gaining a wealth of knowledge for $R_{c u t}$ in a large set of known magnetic materials will also be empirically useful for assessing the relevance of such models.

In this work, based on linear spin-wave theory (LSWT), we find that for a general quadratic spin Hamiltonian, the sum of square of magnon energies (SSME) at arbitrary $k$ point in BZ can be directly obtained by the Fourier transform of MEIs, consequently one can easily calculate SSME at arbitrary $k$ point in BZ without diagonalization. Thus, different from conventional symmetry analysis which groups the magnon energies into symmetry-related $k$ points, our method produces different relationships between the SSME at different highsymmetry $k$ points subjected to different $R_{\text {cut }}$. Thus, using the magnon energies at only several high-symmetry $k$ points, which can be measured by inelastic neutron scattering accurately [1-6], one can unambiguously assert up to which neighbor the MEIs becomes negligible. To demonstrate how our algorithm works, we show an example for Heisenberg model with ferromagnetic (FM) configuration and give the discussion about general cases with DM interaction, single-ion anisotropy (SIA) as well as non-collinear magnetic ordering. Instead of exhaustedly listing the SSME relationships for all magnetic space group (MSG), we provide a code in the Supplemental Materials (SM). With the basic information about a magnetic material (i.e. space group, the positions and magnetic moments orientations of the magnetic ions), the code will deliver corresponding SSME relationships according to the input $R_{\text {cut }}$. Thus through simply checking up to which $R_{\text {cut }}$, the experimental SSME start to deviate from the obtained theoretical relationships, one can determine the real-space range of sizable MEIs. Our method can be easily extended to other Hamiltonian with quadratic Fermi or boson operators, thus is useful for the characteristics of the electronic band structure, phonon spectrum, etc.

\section{METHOD}

Usually the magnetic properties of crystal material$\mathrm{s}$ can be well described by a general pairwise magnetic model [1-6]

$$
H=\sum_{l, n, \alpha, l^{\prime}, n^{\prime}, \beta} J_{\mathbf{R}_{l}+\boldsymbol{\tau}_{n}, \mathbf{R}_{l^{\prime}}+\boldsymbol{\tau}_{n^{\prime}}}^{\alpha, \beta} S_{l n}^{\alpha} S_{l^{\prime} n^{\prime}}^{\beta}
$$

where $J_{\mathbf{R}_{l}+\boldsymbol{\tau}_{n}, \mathbf{R}_{l^{\prime}}+\boldsymbol{\tau}_{n^{\prime}}}^{\alpha, \beta}$, a $3 \times 3$ tensor, represents the spin exchange parameter. Here $\mathbf{R}_{l}$ and $\boldsymbol{\tau}_{n}$ represent the lattice translation vector and the position of magnetic ions in the lattice basis, while $\alpha$ and $\beta$ denote $x, y$ or $z$ the cartesian components. As a $3 \times 3$ real tensor, $\mathbf{J}_{\mathbf{R}_{l}+\boldsymbol{\tau}_{n}, \mathbf{R}_{l^{\prime}}+\boldsymbol{\tau}_{n^{\prime}}}$ could be expanded as three terms, and Eq. (1) could be written as

$$
\begin{aligned}
H= & \sum_{l, n, l^{\prime}, n^{\prime}} J_{\mathbf{R}_{l}+\boldsymbol{\tau}_{n}, \mathbf{R}_{l^{\prime}}+\boldsymbol{\tau}_{n^{\prime}}} \mathbf{S}_{l n} \cdot \mathbf{S}_{l^{\prime} n^{\prime}} \\
& +\sum_{l, n, l^{\prime}, n^{\prime}} \mathbf{D}_{\mathbf{R}_{l}+\boldsymbol{\tau}_{n}, \mathbf{R}_{l^{\prime}}+\boldsymbol{\tau}_{n^{\prime}}} \cdot\left[\mathbf{S}_{l n} \times \mathbf{S}_{l^{\prime} n^{\prime}}\right] \\
& +\sum_{l, n, l^{\prime}, n^{\prime}} \mathbf{S}_{l n} \cdot \Gamma_{\mathbf{R}_{l}+\boldsymbol{\tau}_{n}, \mathbf{R}_{l^{\prime}}+\boldsymbol{\tau}_{n^{\prime}}} \cdot \mathbf{S}_{l^{\prime} n^{\prime}}
\end{aligned}
$$

Here the first term describes the isotropic Heisenberg Hamiltonian with the scalar term $J_{\mathbf{R}_{l}+\boldsymbol{\tau}_{n}, \mathbf{R}_{l^{\prime}}+\boldsymbol{\tau}_{n^{\prime}}}$, the second one represents the antisymmetric DzyaloshinskiiMoriya (DM) interactions with the vector term $\mathbf{D}_{\mathbf{R}_{l}+\boldsymbol{\tau}_{n}, \mathbf{R}_{l^{\prime}}+\boldsymbol{\tau}_{n^{\prime}}}[44,45]$, and the third one is the rest of anisotropic terms with the symmetric tensor term $\Gamma_{\mathbf{R}_{l}+\boldsymbol{\tau}_{n}, \mathbf{R}_{l^{\prime}}+\boldsymbol{\tau}_{n^{\prime}}}$ [45]. It is commonly believed that the magnitude of the DM interaction and $\Gamma_{\mathbf{R}_{l}+\boldsymbol{\tau}_{n}, \mathbf{R}_{l^{\prime}}+\boldsymbol{\tau}_{n^{\prime}}}$ are proportional to spin-orbit coupling (SOC) strength $\lambda$ and $\lambda^{2}$, respectively [45]. For the materials with large $\lambda$, such as $f$ electronic systems, multipolar interactions may become important [46], thus we restrict us on the cases with small $\lambda$ and ignore the third term in Eq. (2) ${ }^{1}$. To take account for non-collinear cases, we use the polar angle $\theta_{n}$ and azimuthal angle $\phi_{n}$ for the spin orientation of magnetic ion at $n$ site.

Following the LSWT [5], we perform the HolsteinPrimakoff transformation and the Fourier transformation, and the Eq. (1) could be written as

$$
\sum_{k} \psi^{\dagger}(\mathbf{k}) H(\mathbf{k}) \psi(\mathbf{k})
$$

\footnotetext{
1 Eq. (1) may also not be suitable for the case with orbitally degenerate [47]
} 
where $\psi^{\dagger}(\mathbf{k})=\left[a_{1}^{\dagger}(\mathbf{k}), \ldots, a_{i}^{\dagger}(\mathbf{k}), \ldots, a_{N}^{\dagger}(\mathbf{k}), a_{1}(-\mathbf{k}), \ldots\right.$, $\left.a_{i}(-\mathbf{k}), \ldots, a_{N}(-\mathbf{k})\right]$, in which $a_{i}^{\dagger}(\mathbf{k})$ and $a_{i}(\mathbf{k})$ represent the canonical boson creation and annihilation operators with wave vector $\mathbf{k}$. Here $i$ runs from 1 to $\mathrm{N}$, and $\mathrm{N}$ is the number magnetic ions per unit cells. The Hermitian matrix $H(\mathbf{k})$ in Eq. (3) is expressed as

$$
H(\mathbf{k})=\left[\begin{array}{cc}
h(\mathbf{k}) & h^{\prime}(\mathbf{k}) \\
h^{\prime}(\mathbf{k})^{\dagger} & h(-\mathbf{k})^{\top}
\end{array}\right]
$$

Here $h(\mathbf{k})$ and $h^{\prime}(\mathbf{k})$ are expressed by

$$
\begin{aligned}
h(\mathbf{k})_{n, n^{\prime}}= & \sum_{l} S\left(A_{n, n^{\prime}} J_{\boldsymbol{\tau}_{n}, \boldsymbol{\tau}_{n^{\prime}}+\mathbf{R}_{l}}+\mathbf{O}_{n, n^{\prime}} \cdot \mathbf{D}_{\boldsymbol{\tau}_{n}, \boldsymbol{\tau}_{n^{\prime}}+\mathbf{R}_{l}}\right) \\
& e^{i \mathbf{k} \cdot \mathbf{R}_{l}}-\delta_{n, n^{\prime}} \sum_{l, n^{\prime \prime}} S\left(B_{n, n^{\prime \prime}} J_{\boldsymbol{\tau}_{n}, \boldsymbol{\tau}_{n^{\prime \prime}}+\mathbf{R}_{l}}\right. \\
& \left.+\mathbf{P}_{n, n^{\prime \prime}} \cdot \mathbf{D}_{\left.\boldsymbol{\tau}_{n}, \boldsymbol{\tau}_{n^{\prime \prime}}+\mathbf{R}_{l}\right)}\right) \\
h^{\prime}(\mathbf{k})_{n, n^{\prime}}= & \sum_{l} S\left(C_{n, n^{\prime}} J_{\boldsymbol{\tau}_{n}, \boldsymbol{\tau}_{n^{\prime}}+\mathbf{R}_{l}}+\mathbf{Q}_{n, n^{\prime}} \cdot \mathbf{D}_{\boldsymbol{\tau}_{n}, \boldsymbol{\tau}_{n^{\prime}}+\mathbf{R}_{l}}\right) \\
& e^{i \mathbf{k} \cdot \mathbf{R}_{l}}
\end{aligned}
$$

where $\delta_{n, n^{\prime}}$ is the Kronecker delta function, while $A_{n, n^{\prime}}, B_{n, n^{\prime}}, C_{n, n^{\prime}}, \mathbf{O}_{n, n^{\prime}}, \mathbf{P}_{n, n^{\prime}}$ and $\mathbf{Q}_{n, n^{\prime}}$ are parameters related to the spin directions at $n$ and $n^{\prime}$ sites (see SM for details).

Considering the commutation relation of $\psi(\mathbf{k})$ and $\psi^{\dagger}(\mathbf{k})$, we need perform the following transformation (see SM for details):

$$
H_{J}(\mathbf{k})=I_{-} H(\mathbf{k})
$$

Through numerically diagonalizing the $H_{J}(\mathbf{k})$ in Eq. (6), we can obtain the magnon energies $\omega_{i}(\mathbf{k})(i=$ $1, \ldots, N)$ at wave vector $k$. In contrary without diagonalization, SSME can be analytically expressed as:

$$
\begin{aligned}
\sum_{i} \omega_{i}^{2}(\mathbf{k})= & \frac{1}{2} \operatorname{Tr}\left(\left[H_{J}(\mathbf{k})\right]^{2}\right) \\
= & \frac{1}{2} \operatorname{Tr}\left[h^{2}(\mathbf{k})+h^{2}(-\mathbf{k})^{\top}-h^{\prime}(\mathbf{k}) h^{\prime}(\mathbf{k})^{\dagger}\right. \\
& \left.-h^{\prime}(\mathbf{k})^{\dagger} h^{\prime}(\mathbf{k})\right]
\end{aligned}
$$

As shown in Eq. (5), $h(\mathbf{k})$ and $h^{\prime}(\mathbf{k})$ basically depend on the orientation of magnetic moments and the Fourier transformation of MEIs $\mathbf{J}_{\mathbf{R}_{l}+\boldsymbol{\tau}_{n}}, \mathbf{R}_{l^{\prime}}+\boldsymbol{\tau}_{n^{\prime}}$ and $\mathbf{D}_{\mathbf{R}_{l}+\boldsymbol{\tau}_{n}, \mathbf{R}_{l^{\prime}}+\boldsymbol{\tau}_{n^{\prime}}}$. Thus, for arbitrary $k, \sum_{i} \omega_{i}^{2}(\mathbf{k})$ can be expressed by a quadratic polynomial of MEIs. With the

\begin{tabular}{|c|c|c|c|c|}
\hline \multirow[t]{2}{*}{$\overline{W P}$} & \multirow[t]{2}{*}{$n$} & \multirow[t]{2}{*}{$\tau_{n}$} & \multicolumn{2}{|c|}{$\left(\theta_{n}, \phi_{n}\right)$} \\
\hline & & & collinear & non-collinear \\
\hline \multirow[t]{4}{*}{$4 d$} & 1 & $(0,0,0)$ & $(0,0)$ & $(\theta, \pi / 2)$ \\
\hline & 2 & $(0.5,0,0)$ & $(0,0)$ & $(\theta,-\pi / 2)$ \\
\hline & 3 & $(0,0.5,0)$ & $(0,0)$ & $(\theta, \pi / 2)$ \\
\hline & 4 & $(0.5,0.5,0)$ & $(0,0)$ & $(\theta,-\pi / 2)$ \\
\hline \multirow[t]{2}{*}{$2 \mathrm{a}$} & 5 & $(0.75,0.25,0)$ & $(0,0)$ & $(0,0)$ \\
\hline & 6 & $(0.25,0.75,0)$ & $(0,0)$ & $(0,0)$ \\
\hline \multirow[t]{2}{*}{$2 \mathrm{c}$} & 7 & $(0.25,0.25,0.1)$ & $(0,0)$ & $(0,0)$ \\
\hline & 8 & $(0.75,0.75,-0.1)$ & $(0,0)$ & $(0,0)$ \\
\hline
\end{tabular}
assumption of $R_{c u t}$, which related with how many MEIs had been considered, one can obtain simple relationships between SSME at different wave vectors $k$.
TABLE I. The WPs and the coordinates of the 8 magnetic ions in the conventional unit cell basis vectors for the example shown here. The Wyckoff positions are labeled in the space group $\mathrm{P} 4 / \mathrm{n}$ (SG 85). The polar angles $\theta_{n}$ and azimuthal

\begin{tabular}{|c|c|c|c|c|}
\hline & distance $(\mathrm{a}$ & & $n^{\prime}$ & $R_{l}$ \\
\hline \multirow[t]{8}{*}{$J_{1}$} & 0.35 & 1 & 5 & $(-1,0,0)$ \\
\hline & & 1 & 6 & $(0,-1,0)$ \\
\hline & & 2 & 5 & $(0,0,0)$ \\
\hline & & 2 & 6 & $(0,-1,0)$ \\
\hline & & 3 & 5 & $(-1,0,0)$ \\
\hline & & 3 & 6 & $(0,0,0)$ \\
\hline & & 4 & 5 & $(0,0,0)$ \\
\hline & & 4 & 6 & $(0,0,0)$ \\
\hline \multirow[t]{8}{*}{$J_{2}$} & 0.36 & 1 & 7 & $(0,0,0)$ \\
\hline & & 1 & 8 & $(-1,-1,0)$ \\
\hline & & 2 & 7 & $(0,0,0)$ \\
\hline & & 2 & 8 & $(0,-1,0)$ \\
\hline & & 3 & 7 & $(0,0,0)$ \\
\hline & & 3 & 8 & $(-1,0,0)$ \\
\hline & & 4 & 7 & $(0,0,0)$ \\
\hline & & 4 & 8 & $(0,0,0)$ \\
\hline \multirow[t]{8}{*}{$J_{3}$} & 0.5 & 1 & 2 & $(0,0,0)$ \\
\hline & & 1 & 2 & $(-1,0,0)$ \\
\hline & & 1 & 3 & $(0,0,0)$ \\
\hline & & 1 & 3 & $(0,-1,0)$ \\
\hline & & 2 & 4 & $(0,0,0)$ \\
\hline & & 2 & 4 & $(0,-1,0)$ \\
\hline & & 3 & 4 & $(0,0,0)$ \\
\hline & & 3 & 4 & $(-1,0,0)$ \\
\hline
\end{tabular}
angles $\phi_{n}$ of these magnetic ions in our selected collinear and non-collinear states are also provided.

TABLE II. The bonds for the 1st, 2nd and 3rd NNs of the example shown in here and the corresponding Heisenberg exchange interactions of the collinear FM configuration (i.e. the case with BNS 85.59). Each bond is characterized by the positions of the two endings: $\boldsymbol{\tau}_{n}, \boldsymbol{\tau}_{n^{\prime}}+\mathbf{R}_{l}$. The unit of distance is taken as the lattice constant $a$.

\section{RESULTS AND DISCUSSION}

We illustrate the usage of our results by following typical example. Without loss of generality, we choose space group $\mathrm{P} 4 / \mathrm{n}$ (SG 85) to present our discussion and set the ratio between lattice constant $c / a$ as 0.8 . We put the magnetic ions at three nonequivalent crystallographic sites: $4 d(0,0,0), 2 a(0.25,0.75,0)$ and $2 c(0.25,0.25$, z) Wyckoff positions (WPs), as summarized in Table I. 
TABLE III. The obtained SSME relationship of the collinear FM example shown in here (i.e. the case with BNS 85.59). The first column $J_{x}$ represents up to the $x$-th NN MEI. The coordinate of six high-symmetry $k$ points: $\Gamma(0,0,0), X\left(\frac{1}{2}, 0,0\right)$, $M\left(\frac{1}{2}, \frac{1}{2}, 0\right), Z\left(0,0, \frac{1}{2}\right), R\left(\frac{1}{2}, 0, \frac{1}{2}\right)$, and $A\left(\frac{1}{2}, \frac{1}{2}, \frac{1}{2}\right)$.

\begin{tabular}{ll}
\hline \hline$J_{\text {max }}$ & relation \\
\hline$J_{2} \quad$ & $\sum_{i} \omega_{i}^{2}(k)=C$ \\
\hline & $\sum_{i} \omega_{i}^{2}(\Gamma)=\sum_{i} \omega_{i}^{2}(Z)$ \\
$J_{4} \quad \sum_{i} \omega_{i}^{2}(X)=\sum_{i} \omega_{i}^{2}(R)$ \\
& $\sum_{i} \omega_{i}^{2}(M)=\sum_{i} \omega_{i}^{2}(A)$ \\
& $2 \sum_{i} \omega_{i}^{2}(X)=\sum_{i} \omega_{i}^{2}(\Gamma)+\sum_{i} \omega_{i}^{2}(M)$ \\
\hline \multirow{3}{*}{$J_{12}$} & $\sum_{i} \omega_{i}^{2}(\Gamma)=\sum_{i} \omega_{i}^{2}(Z)$ \\
& $\sum_{i} \omega_{i}^{2}(X)=\sum_{i}^{2} \omega_{i}^{2}(R)=\sum_{i} \omega_{i}^{2}(A)$ \\
\hline$J_{15}$ & $\sum_{i} \omega_{i}^{2}(\Gamma)-\sum_{i} \omega_{i}^{2}(Z)=\sum_{i} \omega_{i}^{2}(X)-\sum_{i} \omega_{i}^{2}(R)=\sum_{i} \omega_{i}^{2}(M)-\sum_{i} \omega_{i}^{2}(A)$ \\
\hline$J_{22}$ & $2 \sum_{i} \omega_{i}^{2}(X)-\sum_{i} \omega_{i}^{2}(\Gamma)-\sum_{i} \omega_{i}^{2}(M)=2 \sum_{i} \omega_{i}^{2}(R)-\sum_{i} \omega_{i}^{2}(Z)-\sum_{i} \omega_{i}^{2}(A)$ \\
\hline
\end{tabular}

While the $4 d$ and $2 a$ WPs had been completely determined by the spatial symmetry, the coordinates of $2 c \mathrm{WP}$ have a variable $z$ and here we adopt it as $z=0.1$. There are two generators for this space group: the four-fold rotation $\left\{4_{001}^{+} \mid 1 / 2,0,0\right\}$ and inversion operation $\{\overline{1} \mid 0,0,0\}$, where the left part represents the rotation, the right part means the lattice translation, and $\overline{1}$ denotes the inversion symmetry. We firstly consider the most simple case: isotropic Heisenberg model with all the spins along $z$. Considering the orientations of the magnetic moments, the space group could be divided into four types of magnetic space groups ${ }^{2}$. The case with this collinear ferromagnetic (FM) ordering belongs to the type-I magnetic space group (BNS 85.59), and its magnetic configuration does not reduce the spatial symmetry. Since all the spins along $z$ direction, polar angle $\theta_{n}$ and azimuthal angle $\phi_{n}$ are equal to 0 as listed in Table I, thus according to the Eq. (21-23) in SM, the parameters $A_{n, n^{\prime}}, B_{n, n^{\prime}}$ and $C_{n, n^{\prime}}$ in Eq. (5) for this collinear FM state becomes 1, 1 and 0 respectively. Consequently, the SSME at wave vector $k$ could be written as

$$
\begin{aligned}
\sum_{i} \omega_{i}^{2}(\mathbf{k}) & =\frac{1}{2} \operatorname{Tr}\left(\left[H_{J}(\mathbf{k})\right]^{2}\right) \\
& =S^{2} \sum_{n \neq n^{\prime}, l, l^{\prime}} J_{\boldsymbol{\tau}_{n}, \boldsymbol{\tau}_{n^{\prime}}+\mathbf{R}_{l^{\prime}}} J_{\boldsymbol{\tau}_{n}, \boldsymbol{\tau}_{n^{\prime}}+\mathbf{R}_{l}} e^{i \mathbf{k} \cdot\left(\mathbf{R}_{l}-\mathbf{R}_{l^{\prime}}\right)}+S^{2} \sum_{n}\left[\sum_{n^{\prime \prime}, l} J_{\boldsymbol{\tau}_{n}, \boldsymbol{\tau}_{n^{\prime \prime}}+\mathbf{R}_{l}}+\sum_{l} J_{\boldsymbol{\tau}_{n}, \boldsymbol{\tau}_{n}+\mathbf{R}_{l}} e^{i \mathbf{k} \cdot \mathbf{R}_{l}}\right]^{2}
\end{aligned}
$$

As shown in the above formula, the key for SSME is the exchange path between magnetic ions $\boldsymbol{\tau}_{n}$ and $\boldsymbol{\tau}_{n^{\prime}}+\mathbf{R}_{l^{\prime}}$, and the related MEI $J_{\boldsymbol{\tau}_{n}, \boldsymbol{\tau}_{n^{\prime}}+\mathbf{R}_{l^{\prime}}}$. As shown in Table II, the first and second nearest neighbor have similar distances (0.35 $a$ vs $0.36 a, a$ is lattice parameter). Crystal symmetry imposes strong restrictions on the MEIs as shown in SM, and according to the spatial symmetry in this space group, all the first nearest neighbor exchange paths have the same MEI value, and we denotes it as $J_{1}$, same as that, we can label all the second nearest neighbor

\footnotetext{
2 Type-I magnetic space group has no any additional symmetry compared with the corresponding space group, while type-II magnetic space group has an additional anti-symmetry version of every symmetry operation. For type-III magnetic space group, there are additional anti-symmetry versions for the half of its symmetry operations. Specially, type-IV magnetic space group has additional combined spatial translation-time reversal symmetry.
}

MEI as $J_{2}$. Considering only the first two NN interactions $J_{1}$ and $J_{2}$, the term of $J_{\boldsymbol{\tau}_{n}, \boldsymbol{\tau}_{n}+\mathbf{R}_{l}}$ does not exist as shown in Table II, and the only $k$ dependence of SSME comes from the first term in Eq. (8). Namely, we need to check the non-zero MEIs $J_{\boldsymbol{\tau}_{n}, \boldsymbol{\tau}_{n^{\prime}}+\mathbf{R}_{l^{\prime}}}$ and $J_{\boldsymbol{\tau}_{n}, \boldsymbol{\tau}_{n^{\prime}}+\mathbf{R}_{l}}$, with the requirement of $\mathbf{R}_{l} \neq \mathbf{R}_{l^{\prime}}$. As clearly shown in Table II, such kind of exchange path is also not exist. Thus, although spin wave has dispersion at the entire BZ, we get a surprisingly simple result of $\sum_{i} \omega_{i}^{2}(\mathbf{k})=C$ with considering only $J_{1}$ and $J_{2}$.

We further take into account the impact of longerranged exchange paths. With the third NN MEI $J_{3}$ been considered, there exist more than one exchange paths connect a pair of $\boldsymbol{\tau}_{n}$ and $\boldsymbol{\tau}_{n^{\prime}}+\mathbf{R}_{l}$. For example, both $\boldsymbol{\tau}_{1}, \boldsymbol{\tau}_{2}$ pair and $\boldsymbol{\tau}_{1}, \boldsymbol{\tau}_{2}+\mathbf{R}_{-100}$ pair belong to $J_{3}$ exchange path as shown in Table II. As a result, $\sum_{i} \omega_{i}^{2}(\mathbf{k})$ is no longer equal to constant. Thus, if the observed SSME shows very weak $k$ dependence, one can assert$\mathrm{s}$ that the MEIs beyond $J_{2}$ are ignorable. Since for 
the high symmetry $k$ points at BZ, $e^{i \mathbf{k} \cdot\left(\mathbf{R}_{l}-\mathbf{R}_{l^{\prime}}\right)}$ usually has simple values (equals to \pm 1 in this magnetic system), one can expect simple relation between SSME at these $k$ points. We indeed get several simple relationships with the MEIs up to $J_{3}: \sum_{i} \omega_{i}^{2}(\Gamma)=\sum_{i} \omega_{i}^{2}(Z)$, $\sum_{i} \omega_{i}^{2}(X)=\sum_{i} \omega_{i}^{2}(R), \quad \sum_{i} \omega_{i}^{2}(M)=\sum_{i} \omega_{i}^{2}(A)$ and $2 \sum_{i} \omega_{i}^{2}(X)=\sum_{i} \omega_{i}^{2}(\Gamma)+\sum_{i} \omega_{i}^{2}(M)$. It is interest to see that these four simple relations about SSME remains after including the fourth nearest neighbor MEI $J_{4}$. The algorithm about SSME is simple, which allow us quickly analyze the effect of considering further MEI. We summarize the results at Table III. Using the measured magnon energies at only six high-symmetry $k$ points, one can unambiguous determine the real-space range within which the MEI with considerable value based on the Table III.

After collinear FM configuration, we now illustrate the applications for non-collinear case. We still use the crystal structure mentioned above and fix the magnetic moments at $2 a$ and $2 c$ WPs still along $z$ direction. While the azimuthal angle $\phi$ for magnetic moments at $4 d \mathrm{WP}$ is $\pm \pi$, we assume their polar angles as a free parameter $\theta$ as shown in the Table I. Although an isotropic Heisenberg Hamiltonian may not produce the above noncollinearity, we still use it to demonstrate our method and show the discussion about anisotropic spin model at later. This non-collinear magnetic state belongs to the Type-I magnetic space group BNS 13.65. While the inversion symmetry $\{\overline{1} \mid 0,0,0\}$ are maintained, the the deviation from $z$ direction reduces the four-fold rotation symmetry $\left\{4_{001}^{+} \mid 1 / 2,0,0\right\}$ to the two-fold rotation operation $\left\{2_{001} \mid 1 / 2,1 / 2,0\right\}$. As the result, many symmetryrelated exchange pathes in collinear spin ordering case become inequivalent. For example, as shown in Table I of SM, the eight first NN exchange paths in collinear spin ordering are no longer equivalent and divided into two groups, which are labeled as $J_{1}$ and $J_{2}$ for this noncollinear magnetic case. The parameters in Eq. (5) are also depended on the magnetic moment directions, thus non-collinearity results in different relationship between SSME, which are listed in Table IV. We also want to mention that for the localized magnetic systems, the MEIs should not be sensitive to the magnetic configurations as also required by energy-mapping method for calculating MEIs. For such cases, one can still use the symmetry operations in collinear instead of in non-collinear case to determine equivalent exchange path. Namely if this non-collinear magnetism is very localized, the MEIs will still approximately satisfy the Table II. Based on Table II (i.e. the right part of the Table I in SM) instead of the left part of the Table I in SM, we applying our method to this non-collinear case with localized magnetism and list the results in the right part of Table IV. As shown in Table IV, the free parameter $\theta$ about the magnetic moments directions explicitly appear in the relationship about SSME. Thus, for localized non-collinear magnetic materials, one may determine the magnetic moments directions based on the magnon energies at three wave vectors (i.e. $\Gamma, X$ and $M$ ) in the case that MEIs further than $J_{3}$ are ignorable.

It is worth to mention that our method is also valid for the materials with considerable DM interactions [44, 45]. One can still calculate SSME by Eq. (7) and directly use the program provided in SM to explore the relationship between them. The magnetic anisotropy may also comes from the SIA [1-3]. With the SIA considered, the Hamiltonian becomes $H_{\text {total }}=H+H_{S I A}$, here $H$ is the term shown in Eq. (2) while $H_{S I A}$ represents the term of SIA. Here we adopt a popular form $H_{S I A}=\sum_{l, n} K\left(S_{l n}^{z}\right)^{2} \quad[3]$ where $K$ is the strength of SIA. Based on the standard LSWT, one can easily obtain the spin Hamiltonian at arbitrary wave vector $k$ to be $H_{\text {total }}(k)=H_{J}(k)+2 S K I_{-}$. Adding SIA term into the case of Heisenberg model with collinear FM magnetic ordering given in this work, the SSME could be written as

$$
\begin{aligned}
\sum_{i} \omega_{i}^{2}(\mathbf{k})= & \frac{1}{2} \operatorname{Tr}\left[\left(H_{\text {total }}(\mathbf{k})\right)^{2}\right] \\
= & \frac{1}{2} \operatorname{Tr}\left[\left(H_{J}(\mathbf{k})\right)^{2}\right]+4 N S^{2} K^{2}+4 S^{2} K \\
& \sum_{n}\left[\sum_{n^{\prime \prime}, l} J_{\boldsymbol{\tau}_{n}, \boldsymbol{\tau}_{n^{\prime \prime}}+\mathbf{R}_{l}}+\sum_{l} J_{\boldsymbol{\tau}_{n}, \boldsymbol{\tau}_{n}+\mathbf{R}_{l}} e^{i \mathbf{k} \cdot \mathbf{R}_{l}}\right]
\end{aligned}
$$

Based on Eq. (9), one can easily prove that including SIA will not affect the results given in Table III. For the other cases, one can simply use our code which has implemented effect of SIA to obtain the results.

\section{CONCLUSION}

In summary, appropriate magnetic model play crucial role in investigating various magnetic properties. Unfortunately the current methods for extracting MEIs face a severe limitation about how many MEIs need to be included in the spin Hamiltonian. In this work, we circumvent this methodological bottleneck by noticing that for quadratic spin Hamiltonian, there is a simple connection between SSME and the considered MEIs. Namely, there is $R_{c u t}$-related rules between SSME at high-symmetry points. By efficient measurements of magnon energies only at several high-symmetry $k$ points, one can check up to which $R_{c u t}$, the experimental SSME start to deviate from the obtained $R_{c u t}$-related rules, and subsequently determine the real-space range beyond which MEIs can be safely neglected. For the localized non-collinear magnetic systems, our results may also be used to determine the directions of magnetization. We also provide a program, directly utilizing it, one can get the relationship of SSME for any crystal magnetic materials described by Hamiltonian with pairwise spin. Besides the well used symmetry analysis for the symmetry-related $k$ points, we expect that similar generic $R_{c u t}$-sensitive rules also exist 
TABLE IV. The obtained results about SSME for the non-collinear case shown in this work (i.e. the case with symmetry of BNS 13.65). We also give the result for the same non-collinear configuration with the magnetism is very localized in the right part. For the localized magnetism case, the MEIs are not sensitive to the spin ordering, thus one can use the symmetry of SG 85 to determine if the exchange pathes have the same MEIs, namely use the results given in the right part of Table I in SM. The $x$ in $J_{x}$ still represents up to the $x$-th NN MEI, and the coordination of six high-symmetry points had been shown in Table III.

\begin{tabular}{|c|c|}
\hline$J_{\max }$ relation & $J_{\max }$ relation \\
\hline$J_{4} \quad \sum_{i} \omega_{i}^{2}(k)=C$ & $J_{2} \quad \sum_{i} \omega_{i}^{2}(k)=C$ \\
\hline $\begin{array}{l}\sum_{i} \omega_{i}^{2}(\Gamma)=\sum_{i} \omega_{i}^{2}(Z) \\
\sum_{i} \omega_{i}^{2}(X)=\sum_{i} \omega_{i}^{2}(R)=\sum_{i} \omega_{i}^{2}(M)=\sum_{i} \omega_{i}^{2}(A)\end{array}$ & $\begin{array}{ll} & \sum_{i} \omega_{i}^{2}(\Gamma)=\sum_{i} \omega_{i}^{2}(Z) \\
J_{3} & \sum_{i} \omega_{i}^{2}(X)=\sum_{i} \omega_{i}^{2}(R) \\
& \sum_{i} \omega_{i}^{2}(M)=\sum_{i} \omega_{i}^{2}(A) \\
& (1+\cos 2 \theta) \sum_{i} \omega_{i}^{2}(X)=\omega_{i}^{2}(\Gamma)+\cos 2 \theta \sum_{i} \omega_{i}^{2}(M)\end{array}$ \\
\hline $\begin{array}{ll} & \sum_{i} \omega_{i}^{2}(\Gamma)=\sum_{i} \omega_{i}^{2}(Z) \\
J_{24} & \sum_{i} \omega_{i}^{2}(X)=\sum_{i} \omega_{i}^{2}(R) \\
& \sum_{i} \omega_{i}^{2}(M)=\sum_{i} \omega_{i}^{2}(A)\end{array}$ & $\begin{array}{ll}J_{12} \omega_{i}^{2}(\Gamma)=\sum_{i} \omega_{i}^{2}(Z) \\
J_{i} \omega_{i}^{2}(X)=\sum_{i} \omega_{i}^{2}(R) \\
& \sum_{i} \omega_{i}^{2}(M)=\sum_{i} \omega_{i}^{2}(A)\end{array}$ \\
\hline $\begin{array}{c}J_{28} \quad \sum_{i} \omega_{i}^{2}(\Gamma)-\sum_{i} \omega_{i}^{2}(Z)=\sum_{i} \omega_{i}^{2}(X)-\sum_{i} \omega_{i}^{2}(R) \\
=\sum_{i} \omega_{i}^{2}(M)-\sum_{i} \omega_{i}^{2}(A)\end{array}$ & $\begin{array}{c}J_{15} \omega_{i}^{2}(\Gamma)-\sum_{i} \omega_{i}^{2}(Z)=\sum_{i} \omega_{i}^{2}(X)-\sum_{i} \omega_{i}^{2}(R) \\
=\sum_{i} \omega_{i}^{2}(M)-\sum_{i} \omega_{i}^{2}(A)\end{array}$ \\
\hline & $\begin{array}{cc}2 \sum_{i} \omega_{i}^{2}(X)-\sum_{i} \omega_{i}^{2}(\Gamma)-\sum_{i} \omega_{i}^{2}(M) \\
=2 \sum_{i} \omega_{i}^{2}(R)-\sum_{i} \omega_{i}^{2}(Z)-\sum_{i} \omega_{i}^{2}(A)\end{array}$ \\
\hline
\end{tabular}

in other Hamiltonian with only quadratic Fermi or boson operators.

\section{ACKNOWLEDGEMENTS}

This work was supported by the NSFC (No. 11834006 , 12004170, 51721001, and 11790311), National Key R\&D
Program of China (No. 2018YFA0305704 and 2017YFA0303203) and the excellent programme in Nanjing University. Xiangang Wan also acknowledges the support from the Tencent Foundation through the XPLORER PRIZE.
[1] J. Stöhr and H. Siegmann, Magnetism From Fundamentals to Nanoscale Dynamics (Springer, 2006).

[2] K. H. J. Buschow, F. R. Boer, et al., Physics of Magnetism and Magnetic Materials (Springer, 2003).

[3] R. M. White, Quantum Theory of Magnetism: Magnetic Properties of Materials (Springer-Verlag Berlin Heidelberg, 2007).

[4] A. I. Lichtenstein, V. I. Anisimov, and M. I. Katsnelson, Electronic Structure and Magnetism of Correlated Systems: Beyond LDA (Springer, 2003).

[5] A. Prabhakar and D. D. Stancil, Spin waves: Theory and applications, Vol. 5 (Springer, 2009).

[6] M. Krawczyk and D. Grundler, "Review and prospects of magnonic crystals and devices with reprogrammable band structure," J. Phys.: Condens. Matter 26, 123202 (2014).

[7] A. M. Kosevich, B. Ivanov, and A. Kovalev, "Magnetic solitons," Physics Reports 194, 117 (1990).

[8] H. C. Fogedby, "Solitons and magnons in the classical heisenberg chain," Journal of Physics A: Mathematical and General 13, 1467 (1980).

[9] T. Giamarchi, C. Rüegg, and O. Tchernyshyov, "Boseeinstein condensation in magnetic insulators," Nature Physics 4, 198 (2008).

[10] T. Nikuni, M. Oshikawa, A. Oosawa, and H. Tanaka, "Bose-einstein condensation of dilute magnons in tlcucl 3," Phys. Rev. Lett. 84, 5868 (2000).
[11] S. Demokritov, V. Demidov, O. Dzyapko, G. Melkov, A. Serga, B. Hillebrands, and A. Slavin, "Bose-einstein condensation of quasi-equilibrium magnons at room temperature under pumping," Nature 443, 430 (2006).

[12] Y. Onose, T. Ideue, H. Katsura, Y. Shiomi, N. Nagaosa, and Y. Tokura, "Observation of the magnon hall effect," Science 329, 297 (2010).

[13] R. Chisnell, J. Helton, D. E. Freedman, D. Singh, R. Bewley, D. G. Nocera, and Y. S. Lee, "Topological magnon bands in a kagome lattice ferromagnet," Phys. Rev. Lett. 115, 147201 (2015).

[14] H. Kondo, Y. Akagi, and H. Katsura, "Z2 topological invariant for magnon spin hall systems," Phys. Rev. B 99, 041110 (2019).

[15] A. Mook, J. Henk, and I. Mertig, "Edge states in topological magnon insulators," Phys. Rev. B 90, 024412 (2014).

[16] L. Zhang, J. Ren, J.-S. Wang, and B. Li, "Topological magnon insulator in insulating ferromagnet," Phys. Rev. B 87, 144101 (2013).

[17] J. Fransson, A. M. Black-Schaffer, and A. V. Balatsky, "Magnon dirac materials," Phys. Rev. B 94, 075401 (2016).

[18] S. A. Owerre, "Magnonic analogs of topological Dirac semimetals," J. Phys. Commun. 1, 025007 (2017).

[19] N. Okuma, "Magnon Spin-Momentum Locking: Various Spin Vortices and Dirac magnons in Noncollinear Antiferromagnets," Phys. Rev. Lett. 119, 107205 (2017). 
[20] W. Yao, C. Li, L. Wang, S. Xue, Y. Dan, K. Iida, K. Kamazawa, K. Li, C. Fang, and Y. Li, "Topological spin excitations in a three-dimensional antiferromagnet," Nat. Phys. 14, 1011 (2018).

[21] S. Bao, J. Wang, W. Wang, Z. Cai, S. Li, Z. Ma, D. Wang, K. Ran, Z.-Y. Dong, D. L. Abernathy, S.-L. Yu, X. Wan, J.-X. Li, and J. Wen, "Discovery of coexisting Dirac and triply degenerate magnons in a three-dimensional antiferromagnet," Nat. Commun. 9, 2591 (2018).

[22] F.-Y. Li, Y.-D. Li, Y. B. Kim, L. Balents, Y. Yu, and G. Chen, "Weyl magnons in breathing pyrochlore antiferromagnets," Nat. Commun. 7, 12691 (2016).

[23] A. Mook, J. Henk, and I. Mertig, "Tunable magnon Weyl points in ferromagnetic pyrochlores," Phys. Rev. Lett. 117, 157204 (2016).

[24] Y. Su, X. S. Wang, and X. R. Wang, "Magnonic Weyl semimetal and chiral anomaly in pyrochlore ferromagnets," Phys. Rev. B 95, 224403 (2017).

[25] A. Serga, A. Chumak, and B. Hillebrands, "Yig magnonics," Journal of Physics D: Applied Physics 43, 264002 (2010).

[26] V. Kruglyak, S. Demokritov, and D. Grundler, "Magnonics," Journal of Physics D: Applied Physics 43, 264001 (2010).

[27] A. Chumak, V. Vasyuchka, A. Serga, and B. Hillebrands, "Magnon spintronics," Nature Physics 11, 453 (2015).

[28] S. A. Nikitov, D. V. Kalyabin, I. V. Lisenkov, A. Slavin, Y. N. Barabanenkov, S. A. Osokin, A. V. Sadovnikov, E. N. Beginin, M. A. Morozova, Y. A. Filimonov, et al., "Magnonics: a new research area in spintronics and spin wave electronics," Phys.-Usp. 58, 1002 (2015).

[29] B. Lenk, H. Ulrichs, F. Garbs, and M. Münzenberg, "The building blocks of magnonics," Physics Reports 507, 107 (2011).

[30] H. Xiang, C. Lee, H.-J. Koo, X. Gong, and M.H. Whangbo, "Magnetic properties and energy-mapping analysis," Dalton Transactions 42, 823 (2013).

[31] A. I. Liechtenstein, M. I. Katsnelson, V. P. Antropov, and V. A. Gubanov, "Local spin density functional approach to the theory of exchange interactions in ferromagnetic metals and alloys," J. Magn. Magn. Mater. 67, 65 (1987).

[32] P. Bruno, "Exchange Interaction Parameters and Adiabatic Spin-Wave Spectra of Ferromagnets: A Renormalized Magnetic Force Theorem," Phys. Rev. Lett. 90, 085205 (2003).
[33] X. Wan, Q. Yin, and S. Y. Savrasov, "Calculation of magnetic exchange interactions in mott-hubbard systems," Phys. Rev. Lett. 97, 266403 (2006).

[34] H. Ebert, D. Koedderitzsch, and J. Minar, "Calculating condensed matter properties using the kkr-green's function methodrecent developments and applications," Rep. Prog. Phys. 74, 096501 (2011).

[35] A. Secchi, A. I. Lichtenstein, and M. I. Katsnelson, "Magnetic interactions in strongly correlated systems: Spin and orbital contributions," Annals of Physics 360, 61 (2015).

[36] N. M. Rosengaard and B. Johansson, "Finitetemperature study of itinerant ferromagnetism in fe, co, and ni," Physical Review B 55, 14975 (1997).

[37] S. Halilov, H. Eschrig, A. Perlov, and P. Oppeneer, "Adiabatic spin dynamics from spin-density-functional theory: Application to fe, co, and ni," Physical Review B 58, 293 (1998).

[38] J. A. M. Paddison, "Scattering signatures of bonddependent magnetic interactions," Physical Review Lett. 125, 247202 (2020).

[39] V. Anisimov, F. Aryasetiawan, and A. Lichtenstein, "Calculation of magnetic exchange interactions in motthubbard systems," J. Phys.: Condens. Matter 9, 767 (1997).

[40] G. Kotliar, S. Y. Savrasov, K. Haule, V. S. Oudovenko, O. Parcollet, and C. A. Marianetti, "Electronic structure calculations with dynamical mean-field theory," Rev. Mod. Phys. 78, 865 (2006).

[41] P. C. Hohenberg and W. F. Brinkman, "Sum rules for the frequency spectrum of linear magnetic chains," Phys. Rev. B 10, 128 (1974).

[42] A. Kitaev, "Anyons in an exactly solved model and beyond," Annals of Physics 321, 2 (2006).

[43] J. S. Gardner, M. J. Gingras, and J. E. Greedan, "Magnetic pyrochlore oxides," Rev. Mod. Phys. 82, 53 (2010).

[44] I. Dzyaloshinsky, "A thermodynamic theory of "weak" ferromagnetism of antiferromagnetics," J. Phys. Chem. Solids 4, 241 (1958).

[45] T. Moriya, "Anisotropic superexchange interaction and weak ferromagnetism," Physical Review 120, 91 (1960).

[46] P. Santini, S. Carretta, G. Amoretti, R. Caciuffo, N. Magnani, and G. H. Lander, "Multipolar interaction$\mathrm{s}$ in f-electron systems: The paradigm of actinide dioxides," Rev. Mod. Phys. 81, 807 (2009).

[47] K. Kugel and D. Khomskii, "The jahn-teller effect and magnetism: transition metal compounds," Sov. Phys. Usp. 25, 231 (1982). 


\section{Supplementary Files}

This is a list of supplementary files associated with this preprint. Click to download.

- sumrulev36sm.pdf 